\title{
Comparison of Coronary Plaque Rupture Between Stable Angina and Acute Myocardial Infarction A Three-Vessel Intravascular Ultrasound Study in 235 Patients
}

\author{
Myeong-Ki Hong, MD, PhD; Gary S. Mintz, MD; Cheol Whan Lee, MD, PhD; \\ Young-Hak Kim, MD; Seung-Whan Lee, MD; Jong-Min Song, MD, PhD; \\ Ki-Hoon Han, MD; Duk-Hyun Kang, MD, PhD; Jae-Kwan Song, MD, PhD; \\ Jae-Joong Kim, MD, PhD; Seong-Wook Park, MD, PhD; Seung-Jung Park, MD, PhD
}

Background-We evaluated the incidence and predictors of single and multiple plaque ruptures in acute myocardial infarction (AMI) and stable angina pectoris (SAP).

Methods and Results-We performed 3-vessel intravascular ultrasound (IVUS) examination in 235 patients: 122 had AMI, and 113 had SAP. Plaque rupture of infarct-related or target lesions occurred in 80 AMI patients (66\%) and in 31 SAP patients $(27 \%)(P<0.001)$. Non-infarct-related or non-target artery plaque ruptures occurred in 21 AMI patients $(17 \%)$ and 6 SAP patients $(5 \%)(P=0.008)$. Multiple plaque ruptures were observed in 24 AMI $(20 \%)$ and 7 SAP patients $(6 \%)(P=0.004)$. Therefore, at least 1 plaque rupture in any coronary artery was noted in 84 AMI patients (69\%) and 35 SAP patients $(31 \%)$ $(P<0.001)$. Overall, the only independent clinical predictor of plaque rupture in the infarct-related/target lesion was AMI $(P<0.01$; OR, 4.867; 95\% CI, 2.734 to 8.661). The only independent clinical predictor of plaque rupture in AMI patients was an elevated C-reactive protein (CRP) level ( $P=0.035$; OR, 2.139; 95\% CI, 1.053 to 4.343). Conversely, in SAP patients, the only independent clinical predictor of plaque rupture was diabetes mellitus $(P=0.034 ; \mathrm{OR}, 2.553 ; 95 \% \mathrm{CI}, 1.071$ to 6.085$)$. The only independent clinical predictor of multiple plaque ruptures was AMI ( $P=0.003$; OR, 3.752; 95\% CI, 1.546 to 9.105$)$.

Conclusions - Three-vessel IVUS imaging showed that culprit lesion plaque rupture, secondary remote plaque ruptures, and multiple plaque ruptures were all more common in AMI patients than SAP patients. In AMI patients, plaque rupture was associated with a high CRP level, whereas in SAP patients, plaque rupture was more common in those with diabetes. (Circulation. 2004;110:928-933.)

Key Words: ultrasonics $\square$ plaque $\mathbf{a}$ coronary artery disease

$\mathrm{P}$ athological and autopsy studies have reported that rupture of a vulnerable plaque and subsequent thrombus formation is the most important mechanism leading to an acute coronary syndrome (ACS). ${ }^{1,2}$ One previous study using coronary angiography showed that $40 \%$ of patients with an acute myocardial infarction (AMI) had multiple complex plaques and that these patients had an increased incidence of recurrent ACS, repeat intervention, and coronary-artery bypass surgery in the subsequent year. ${ }^{3}$ Intravascular ultrasound (IVUS) provides detailed, high-quality tomographic images of coronary plaque and vessel wall and can safely detect plaque rupture in vivo. IVUS plaque rupture is typically associated with an angiographically complex-appearing plaque; however, secondary plaque ruptures are usually missed if they occur in the same artery. ${ }^{4}$ Other IVUS studies have evaluated the incidence and clinical presentation of single and multiple plaque ruptures in patients with stable angina pectoris (SAP), ACS, or AMI. ${ }^{5-8}$ To date, the only 3-vessel
IVUS study in ACS patients reported an incidence of culprit lesion plaque rupture of $37.5 \%$ (9 of 24), but $79 \%$ (19 of 24) of the patients had at least 1 secondary (nonculprit) plaque rupture. ${ }^{6}$ These data have profound implications on strategies to identify vulnerable plaques, in particular, multiple vulnerable ruptureprone plaques, in patients at risk for an AMI. Therefore, to confirm these data, we performed a prospective IVUS analysis of all 3 major epicardial arteries in a large number of AMI patients and compared them with those of a control population of SAP patients. Our goal was to evaluate the incidence and predictors of single and multiple plaque ruptures in AMI and SAP patients.

\section{Methods}

\section{Study Population}

Between July 2002 and April 2003, a prospective but nonconsecutive series of 235 patients who were scheduled for coronary intervention

Received December 10, 2003; de novo received February 25, 2004; revision received April 20, 2004; accepted April 22, 2004.

From the Department of Medicine, University of Ulsan College of Medicine, Asan Medical Center, Seoul, Korea; and the Cardiovascular Research Foundation, New York, NY (G.S.M.).

Correspondence to Seung-Jung Park, MD, PhD, Departments of Medicine, University of Ulsan College of Medicine, Asan Medical Center, 388-1 Poongnap-dong, Songpa-gu, Seoul, 138-736, Korea. E-mail sjpark@amc.seoul.kr

(C) 2004 American Heart Association, Inc. 
TABLE 1. Baseline Characteristics of Patients With 3-Vessel IVUS, 1- or 2-Vessel IVUS, and Without IVUS

\begin{tabular}{|c|c|c|c|c|}
\hline & $\begin{array}{l}\text { 3-Vessel } \\
\text { IVUS }\end{array}$ & $\begin{array}{l}\text { 1- or } 2 \text {-Vessel } \\
\text { IVUS }\end{array}$ & $\begin{array}{l}\text { No } \\
\text { IVUS }\end{array}$ & $P$ \\
\hline \multicolumn{5}{|l|}{ Acute myocardial infarction } \\
\hline No. of patients & 122 & 63 & 122 & \\
\hline Age, y & $58 \pm 11$ & $58 \pm 9$ & $59 \pm 15$ & 0.3 \\
\hline Male & $91(75)$ & $48(76)$ & $88(72)$ & 0.8 \\
\hline Hypertension & $45(37)$ & $27(43)$ & $56(46)$ & 0.4 \\
\hline Diabetes mellitus & $17(14)$ & $15(24)$ & $26(21)$ & 0.18 \\
\hline Cigarette smoking & $60(49)$ & $32(51)$ & $53(43)$ & 0.5 \\
\hline $\begin{array}{l}\text { Hypercholesterolemia (total cholesterol } \\
\geq 220 \mathrm{mg} / \mathrm{dL} \text { ) }\end{array}$ & $18(15)$ & $5(8)$ & $14(12)$ & 0.4 \\
\hline No. of diseased vessels & & & & 0.002 \\
\hline 1 & $77(63)$ & $26(41)$ & $47(39)$ & \\
\hline 2 & $29(24)$ & $22(35)$ & $44(36)$ & \\
\hline 3 & $16(13)$ & $15(24)$ & $31(25)$ & \\
\hline \multicolumn{5}{|l|}{ Stable angina pectoris } \\
\hline No. of patients & 113 & 182 & 202 & \\
\hline Age, y & $58 \pm 9$ & $59 \pm 13$ & $59 \pm 12$ & 0.4 \\
\hline Male & $79(70)$ & $131(72)$ & $145(72)$ & 0.9 \\
\hline Hypertension & $46(41)$ & $84(47)$ & $93(46)$ & 0.6 \\
\hline Diabetes mellitus & $34(30)$ & $45(25)$ & $65(32)$ & 0.3 \\
\hline Cigarette smoking & $54(48)$ & $75(41)$ & $91(45)$ & 0.5 \\
\hline $\begin{array}{l}\text { Hypercholesterolemia (total cholesterol } \\
\geq 220 \mathrm{mg} / \mathrm{dL} \text { ) }\end{array}$ & $12(11)$ & $16(9)$ & $21(10)$ & 0.8 \\
\hline No. of diseased vessels & & & & 0.010 \\
\hline 1 & $71(62)$ & $101(55)$ & $92(45)$ & \\
\hline 2 & $29(26)$ & $47(26)$ & $56(28)$ & \\
\hline 3 & $13(12)$ & $34(19)$ & $54(27)$ & \\
\hline
\end{tabular}

underwent 3-vessel IVUS examination. Patients with a history of myocardial infarction, long lesions (length $>30 \mathrm{~mm}$ ), total occlusions, and severe angulation or calcification in any major epicardial artery were excluded (that is, 3-vessel IVUS was not even attempted) because of the potential difficulty in performing and interpreting IVUS in such situations. The study population consisted of 113 SAP and 122 AMI patients. Coronary intervention was performed in 497 SAP and 307 AMI patients during the same study period. IVUSguided intervention was performed in 295 (59\%) of the 497 SAP and $185(60 \%)$ of the 307 AMI patients. Baseline clinical characteristics of patients without IVUS examination, with 1- or 2-vessel IVUS, and with 3-vessel IVUS examination are shown in Table 1.

AMI was defined as continuous chest pain at rest with abnormal levels of cardiac enzymes (creatinine kinase-MB or troponin-T); 91 patients had ST-segment elevation $(>0.1 \mathrm{mV}$ in 2 contiguous ECG leads), and 31 patients did not. Seventy-one of 91 patients with ST-segment elevation underwent primary stenting within 12 hours of symptom onset. Ten patients with ST-segment elevation who underwent primary thrombolytic treatment, 10 patients with ST-segment elevation who arrived at the emergency room $>12$ hours after symptom onset, and 31 patients without ST-segment elevation underwent elective stenting. Overall, the mean duration from AMI onset to IVUS was $1.4 \pm 1.9$ days. Platelet glycoprotein IIb/IIIa receptor inhibitors were used in 22 patients $(18 \%)$. Multivessel interventions were performed in 34 AMI patients (28\%).

SAP was defined as no change in frequency, duration, or intensity of symptoms within 6 weeks before the intervention. ${ }^{9}$ None of the SAP patients received platelet glycoprotein IIb/IIIa receptor inhibi- tors. Multivessel interventions were performed in 29 SAP patients (26\%).

The infarct-related or target lesion was identified by the combination of left ventricular wall motion abnormalities, ECG findings, angiographic lesion morphology, and scintigraphic defects. In SAP patients treated with multivessel intervention, lesions with more severe diameter stenosis and more complex lesion morphology in the vessel territory of scintigraphic reversible defects were selected as the target lesions.

Serum samples were collected just before coronary intervention and IVUS (1.4 \pm 1.9 days after symptom onset). C-reactive protein (CRP) was measured by use of a high-sensitivity turbidimetric assay with a coefficient of variation of $<5 \%$ (hs-CRP, Cobas Integra, Roche Diagnostics). The low detection threshold of this method is $0.0064 \mathrm{mg} / \mathrm{dL} .^{10}$

Other standard risk factors were assessed. Diabetic patients were defined as those undergoing treatment with oral hypoglycemic agents or insulin at study entry.

\section{IVUS Imaging and Analysis}

IVUS examinations of all 3 major epicardial arteries were performed before any intervention and after intracoronary administration of 0.2 $\mathrm{mg}$ nitroglycerin using a motorized transducer pullback system $(0.5 \mathrm{~mm} / \mathrm{s})$ and a commercial scanner (Boston Scientific Corp/ SCIMED) consisting of a rotating $30-\mathrm{MHz}$ transducer within a $3.2 \mathrm{~F}$ imaging sheath. Qualitative and quantitative analyses were performed according to criteria of the American College of Cardiology's Clinical Expert Consensus Document on IVUS. ${ }^{11}$ 


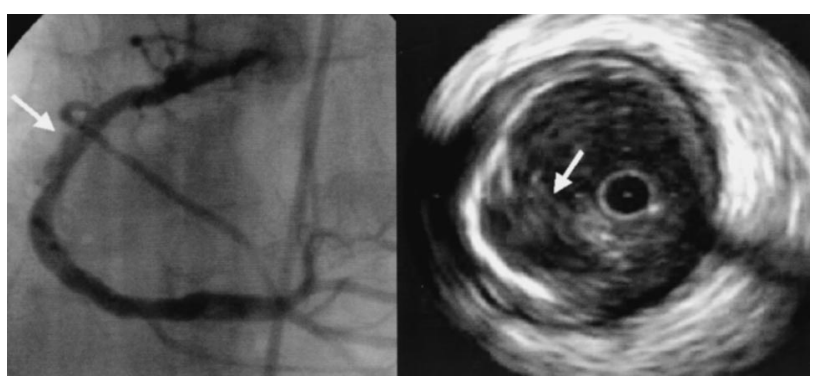

Figure 1. Angiographic and IVUS images of typical appearance of ruptured plaque (arrow) at proximal right coronary artery.

IVUS signs of plaque rupture suggested that a ruptured plaque contained a cavity that communicated with the lumen with an overlying residual fibrous cap fragment. ${ }^{7}$ The diagnosis of plaque rupture required independent review and agreement by 2 of the authors (M.-K.H. and Y.-H.K.). Figure 1 shows a typical plaque rupture. A fissure without a cavity communicating with the true lumen was not included in the analysis. Identification of 2 separate plaques in the same artery (ie, infarct-related/target lesion versus non-infarct-related/target lesion) required a $\geq 5$-mm reference segment between them; if not, they were considered to be part of one long lesion. ${ }^{7}$ The intraplaque cavity was measured and extrapolated to the ruptured capsular area. ${ }^{6}$

Quantitative IVUS analysis was performed by use of computerized planimetry at the infarct-related or target lesion and proximal and distal reference segments. The reference segments were the most normal-looking cross sections within $5 \mathrm{~mm}$ proximal and distal to the lesion but before any side branch. Quantitative measurements included external elastic membrane (EEM), lumen, and plaque and media ( $\mathrm{P}$ and $\mathrm{M}=\mathrm{EEM}$ minus lumen) cross-sectional area (CSA). A remodeling index was calculated as the lesion EEM CSA divided by the mean reference EEM CSA. Expansive (or positive) remodeling was defined as a remodeling index $>1.0 .^{11}$

\section{Quantitative Coronary Angiographic Analysis}

Using the guiding catheter for magnification-calibration and an online system (ANCOR V2.0, Siemens), minimal luminal diameters (MLDs) of the infarct-related/target-lesion-segment and referencesegment lumen diameters were measured before coronary intervention.

\section{Statistical Analysis}

Statistical analysis was performed with SPSS program. Data are presented as frequencies or mean $\pm \mathrm{SD}$. Comparison was performed with a Pearson's $\chi^{2}$ or Fisher's exact test and unpaired Student's $t$ test or ANOVA. Multivariate logistic regression analysis was performed to assess independent clinical predictors for plaque rupture of the infarct-related/target lesion or multiple plaque ruptures. A probability value of $P<0.05$ was considered statistically significant.

\section{Results}

Baseline clinical characteristics and infarct-related or target lesion quantitative coronary angiographic (QCA) and IVUS measurements of all AMI and SAP patients are shown in Table 2. The group with SAP included more patients with diabetes, whereas total cholesterol and CRP level were significantly higher in AMI patients. QCA reference diameter was significantly larger in AMI patients compared with SAP patients. IVUS measurements showed that proximal reference EEM and lumen CSA and lesion site EEM CSA were also significantly larger in AMI patients along with more expansive lesion site remodeling.
TABLE 2. Baseline Clinical Characteristics of All Patients and QCA and IVUS Measurements of Infarct-Related/Target Lesions

\begin{tabular}{|c|c|c|c|}
\hline & $\begin{array}{c}\text { SAP } \\
\text { Patients }\end{array}$ & $\begin{array}{c}\text { AMI } \\
\text { Patients }\end{array}$ & $P$ \\
\hline No. of patients & 113 & 122 & \\
\hline Age, y & $58 \pm 9$ & $58 \pm 11$ & 0.5 \\
\hline Male & $79(70)$ & $91(75)$ & 0.5 \\
\hline Hypertension & $46(41)$ & $45(37)$ & 0.6 \\
\hline Diabetes mellitus & $34(30)$ & $17(14)$ & 0.005 \\
\hline Cigarette smoking & $54(48)$ & $60(49)$ & 0.9 \\
\hline \multicolumn{4}{|l|}{ Lipid profiles } \\
\hline Total cholesterol, mg/dL & $177 \pm 33$ & $191 \pm 35$ & 0.002 \\
\hline LDL cholesterol, mg/dL & $106 \pm 30$ & $117 \pm 30$ & 0.008 \\
\hline Triglyceride, mg/dL & $149 \pm 83$ & $157 \pm 113$ & 0.6 \\
\hline HDL cholesterol, mg/dL & $42 \pm 9$ & $43 \pm 10$ & 0.4 \\
\hline CRP level, mg/dL & $0.5 \pm 0.8$ & $0.9 \pm 1.3$ & 0.004 \\
\hline No. of diseased vessels & & & 0.9 \\
\hline 1 & $71(62)$ & $77(63)$ & \\
\hline 2 & $29(26)$ & $29(24)$ & \\
\hline 3 & $13(12)$ & $16(13)$ & \\
\hline \multicolumn{4}{|l|}{ QCA measurements } \\
\hline Reference segment, mm & $3.2 \pm 0.4$ & $3.4 \pm 0.6$ & 0.026 \\
\hline MLD, mm & $0.7 \pm 0.4$ & $0.6 \pm 0.6$ & 0.2 \\
\hline \multicolumn{4}{|l|}{ IVUS measurements } \\
\hline \multicolumn{4}{|l|}{ Proximal reference segment } \\
\hline EEM CSA, mm² & $14.3 \pm 3.4$ & $15.7 \pm 3.8$ & 0.003 \\
\hline Lumen CSA, mm² & $8.5 \pm 2.0$ & $9.5 \pm 2.4$ & 0.001 \\
\hline \multicolumn{4}{|l|}{ Lesion segment } \\
\hline Plaque cavity CSA, mm² & $1.8 \pm 1.3$ & $2.0 \pm 2.2$ & 0.3 \\
\hline EEM CSA, mm² & $13.3 \pm 3.7$ & $15.5 \pm 4.0$ & $<0.001$ \\
\hline Lumen CSA, mm² & $2.2 \pm 0.3$ & $2.2 \pm 0.5$ & 0.9 \\
\hline Expansive remodeling, \% & $52(46)$ & $83(68)$ & 0.001 \\
\hline Calcium arc, ${ }^{\circ}$ & $50 \pm 70$ & $43 \pm 66$ & 0.4 \\
\hline Distal reference segment & & & \\
\hline EEM CSA, $\mathrm{mm}^{2}$ & $12.4 \pm 3.7$ & $13.1 \pm 3.4$ & 0.14 \\
\hline Lumen CSA, mm² & $7.6 \pm 2.4$ & $7.9 \pm 2.2$ & 0.4 \\
\hline
\end{tabular}

Infarct-related lesion plaque rupture was found in $80 \mathrm{AMI}$ patients $(66 \%)$, and target lesion plaque rupture occurred in 31 SAP patients $(27 \%)(P<0.001)$. There was at least 1 non-infarct-related artery plaque rupture in 21 AMI patients $(17 \%)$, and in 6 SAP patients, there was at least 1 plaque rupture in a nontarget artery $(5 \%)(P=0.008)$. Multiple plaque ruptures were observed in 24 AMI patients (20\%) and 7 SAP patients $(6 \%)(P=0.004)$. Therefore, at least 1 plaque rupture in any coronary artery was noted in 84 AMI patients $(69 \%)$ and 35 SAP patients $(31 \%)(P<0.001)$ (Figure 2$)$. The EEM, lumen, and ruptured-plaque cavity CSA were $16.3 \pm 4.9,5.4 \pm 2.4$, and $2.0 \pm 0.7 \mathrm{~mm}^{2}$ in non-infarct-related ruptured plaque and $16.5 \pm 6.4,5.7 \pm 2.6$, and $1.9 \pm 1.2 \mathrm{~mm}^{2}$ in nontarget ruptured plaque, respectively.

In AMI patients, the incidence of infarct-related lesions and secondary plaque ruptures were similar among the 3 different subgroups (Table 3). 


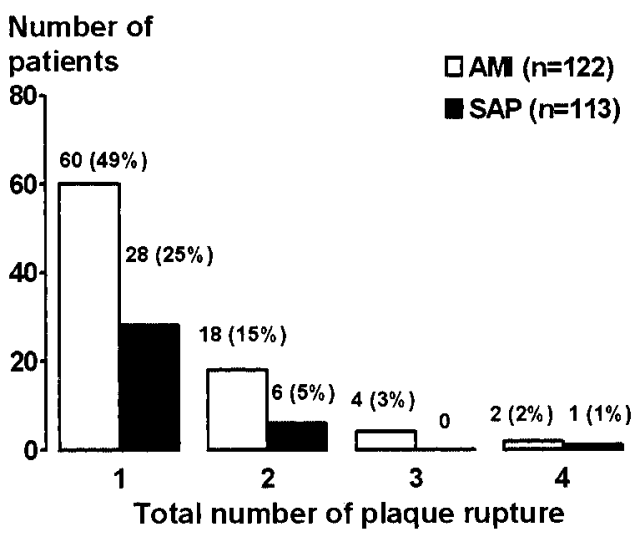

Figure 2. Frequency of plaque rupture between $\mathrm{AMI}$ and SAP patients.

Clinical characteristics, QCA measurements, and IVUS findings comparing SAP patients with and without target lesion plaque rupture are shown in Table 4 . The group with SAP and plaque rupture had more diabetes, larger QCA reference diameters, and larger IVUS reference EEM and lumen CSA and lesion site EEM CSA than the group with SAP and no plaque rupture. A similar analysis of AMI patients showed that those with plaque rupture had higher CRP levels, larger IVUS reference and lesion site EEM CSA, more expansive lesion site remodeling, and larger QCA and IVUS reference lumen dimensions (Table 5).

Multivariate logistic regression analysis was performed to determine the independent clinical predictors of infarctrelated or target lesion plaque rupture. All clinical variables with a value of $P<0.2$ in univariate analysis were tested. In the entire cohort of 235 patients, the only independent predictor of plaque rupture was AMI $(P<0.01$; OR, 4.867; 95\% CI, 2.734 to 8.661). In AMI patients, the only independent predictor of plaque rupture was elevated CRP level $(P=0.035$; OR, 2.139; $95 \% \mathrm{CI}, 1.053$ to 4.343$)$. In SAP patients, the only independent predictor was diabetes mellitus $(P=0.034$; OR, 2.553; 95\% CI, 1.071 to 6.085$)$. The only independent predictor of multiple plaque ruptures was AMI $(P=0.003$; OR, 3.752; 95\% CI, 1.546 to 9.105$)$.

\section{Discussion}

In this prospective analysis of 235 patients who underwent 3-vessel IVUS examination, we found that primary (infarct-
TABLE 4. Baseline Clinical Characteristics and QCA and IVUS Measurements of Target Lesions Comparing SAP Patients With and Without Plaque Rupture

\begin{tabular}{|c|c|c|c|}
\hline & $\begin{array}{l}\text { Plaque } \\
\text { Rupture }\end{array}$ & $\begin{array}{l}\text { No Plaque } \\
\text { Rupture }\end{array}$ & $P$ \\
\hline No. & 31 & 82 & \\
\hline Age, y & $58 \pm 9$ & $59 \pm 10$ & 0.6 \\
\hline Hypertension & $13(42)$ & $33(40)$ & 1.0 \\
\hline Diabetes mellitus & $14(45)$ & $20(24)$ & 0.032 \\
\hline Cigarette smoking & $13(42)$ & $41(50)$ & 0.6 \\
\hline \multicolumn{4}{|l|}{ Lipid profiles } \\
\hline Total cholesterol, mg/dL & $180 \pm 32$ & $177 \pm 33$ & 0.7 \\
\hline LDL cholesterol, mg/dL & $109 \pm 26$ & $105 \pm 32$ & 0.6 \\
\hline Triglyceride, mg/dL & $150 \pm 79$ & $148 \pm 85$ & 0.9 \\
\hline HDL cholesterol, mg/dL & $40 \pm 9$ & $42 \pm 9$ & 0.4 \\
\hline CRP level, mg/dL & $0.6 \pm 1.3$ & $0.4 \pm 0.5$ & 0.3 \\
\hline \multicolumn{4}{|l|}{ QCA } \\
\hline Reference segment, mm & $3.3 \pm 0.5$ & $3.2 \pm 0.4$ & 0.059 \\
\hline MLD, mm & $0.7 \pm 0.4$ & $0.7 \pm 0.3$ & 0.4 \\
\hline \multicolumn{4}{|l|}{ IVUS } \\
\hline \multicolumn{4}{|l|}{ Proximal reference } \\
\hline EEM CSA, $\mathrm{mm}^{2}$ & $16.5 \pm 4.5$ & $13.5 \pm 2.6$ & 0.002 \\
\hline Lumen CSA, mm² & $9.5 \pm 2.3$ & $8.1 \pm 1.7$ & 0.008 \\
\hline \multicolumn{4}{|l|}{ Lesion } \\
\hline EEM CSA, mm² & $15.4 \pm 4.4$ & $12.5 \pm 3.1$ & 0.002 \\
\hline Lumen CSA, mm² & $2.3 \pm 0.3$ & $2.2 \pm 0.3$ & 0.18 \\
\hline Expansive remodeling, \% & $15(49)$ & $37(45)$ & 0.5 \\
\hline Calcium arc, ${ }^{\circ}$ incrict & $44 \pm 66$ & $67 \pm 79$ & 0.11 \\
\hline \multicolumn{4}{|l|}{ Distal reference Asst } \\
\hline EEM CSA, $\mathrm{mm}^{2}$ & $13.9 \pm 4.5$ & $11.8 \pm 3.2$ & 0.023 \\
\hline Lumen CSA, mm² & $8.4 \pm 2.8$ & $7.4 \pm 2.2$ & 0.051 \\
\hline
\end{tabular}

related or target lesion) and secondary plaque rupture occurred not only in AMI patients but also in some SAP patients; however, their frequency was lower in SAP than in AMI patients. The findings of the present study contrast sharply with the only other published 3 -vessel IVUS study. ${ }^{6}$ The present study found a higher incidence of infarct-related lesion plaque rupture $(66 \%$ versus $37.5 \%)$ but a lower incidence of multiple plaque ruptures (20\% versus $79 \%)$.

TABLE 3. Incidence of Plaque Ruptures in AMI Patients

\begin{tabular}{lccc}
\hline & $\begin{array}{c}\text { AMl With } \\
\text { AMI With } \\
\text { ST-Segment } \\
\text { Elevation and } \\
\text { Primary Stenting }\end{array}$ & $\begin{array}{c}\text { ST-Segment } \\
\text { Elevation and } \\
\text { Elective } \\
\text { Stenting }\end{array}$ & $\begin{array}{r}\text { AMI Without } \\
\text { ST-Segment } \\
\text { Elevation }\end{array}$ \\
\hline No. & 71 & 20 & 31 \\
Infarct-related artery & & & $22(71)$ \\
\multicolumn{1}{c}{ Infarct-related lesion } & $44(62)$ & $14(70)$ & $1(3)$ \\
Non-infarct-related lesion & $6(9)$ & $3(15)$ & $5(16)$ \\
Non-infarct-related arteries & $13(18)$ & $3(15)$ & $4(13)$ \\
Multiple plaque rupture & $17(24)$ & $16(80)$ & $23(74)$ \\
\hline
\end{tabular}


TABLE 5. Baseline Clinical Characteristics and QCA and IVUS Measurements of Infarct-Related Lesions Comparing AMI Patients With and Without Plaque Rupture

\begin{tabular}{|c|c|c|c|}
\hline & $\begin{array}{l}\text { Plaque } \\
\text { Rupture }\end{array}$ & $\begin{array}{l}\text { No Plaque } \\
\text { Rupture }\end{array}$ & $P$ \\
\hline No. & 80 & 42 & \\
\hline Age, y & $59 \pm 11$ & $57 \pm 10$ & 0.3 \\
\hline Hypertension & $30(38)$ & $15(36)$ & 1.0 \\
\hline Diabetes mellitus & $12(15)$ & $5(12)$ & 0.8 \\
\hline Cigarette smoking & $41(51)$ & $19(45)$ & 0.6 \\
\hline \multicolumn{4}{|l|}{ Lipid profiles } \\
\hline Total cholesterol, mg/dL & $194 \pm 37$ & $187 \pm 31$ & 0.3 \\
\hline LDL cholesterol, mg/dL & $117 \pm 31$ & $118 \pm 27$ & 0.9 \\
\hline Triglyceride, mg/dL & $171 \pm 130$ & $131 \pm 69$ & 0.070 \\
\hline HDL cholesterol, mg/dL & $43 \pm 10$ & $43 \pm 11$ & 0.6 \\
\hline CRP level, mg/dL & $1.0 \pm 1.5$ & $0.5 \pm 0.6$ & 0.007 \\
\hline \multicolumn{4}{|l|}{ QCA } \\
\hline Reference segment, mm & $3.5 \pm 0.5$ & $3.2 \pm 0.6$ & 0.004 \\
\hline MLD, mm & $0.6 \pm 0.6$ & $0.5 \pm 0.6$ & 0.5 \\
\hline \multicolumn{4}{|l|}{ IVUS } \\
\hline \multicolumn{4}{|l|}{ Proximal reference } \\
\hline EEM CSA, mm² & $16.4 \pm 3.6$ & $14.3 \pm 3.9$ & 0.004 \\
\hline Lumen CSA, mm² & $10.0 \pm 2.3$ & $8.5 \pm 2.4$ & 0.001 \\
\hline \multicolumn{4}{|l|}{ Lesion } \\
\hline EEM CSA, mm² & $16.2 \pm 3.6$ & $14.2 \pm 4.4$ & 0.011 \\
\hline Lumen CSA, mm² & $2.2 \pm 0.4$ & $2.2 \pm 0.7$ & 1.0 \\
\hline Expansive remodeling, \% & $59(74)$ & $24(57)$ & 0.048 \\
\hline Calcium arc, ${ }^{\circ}$ & $35 \pm 60$ & $57 \pm 73$ & 0.11 \\
\hline \multicolumn{4}{|l|}{ Distal reference } \\
\hline EEM CSA, mm² & $13.6 \pm 3.1$ & $12.0 \pm 3.7$ & 0.008 \\
\hline Lumen CSA, mm² & $8.3 \pm 2.0$ & $7.3 \pm 2.5$ & 0.017 \\
\hline
\end{tabular}

Retrospective pathological studies of patients with coronary artery disease who died suddenly showed culprit lesion plaque rupture in $\approx 70 \%$ of patients. ${ }^{1,2,12,13}$ IVUS studies have reported varying frequencies of infarct-related plaque rupture in AMI patients: $15.8 \%$ by Kotani et al, ${ }^{7} 37.5 \%$ by Rioufol et al, ${ }^{6} 37 \%$ by Fukuda et al, ${ }^{8}$ and $55.6 \%$ by Sano et al. ${ }^{14}$ The time from symptom onset to IVUS imaging was $<7$ days, $^{7}$ $<4$ weeks, ${ }^{6}<10$ hours, ${ }^{8}$ and $<6$ hours, ${ }^{14}$ respectively. In the present study, plaque rupture was observed in $66 \%$ of AMI patients; the time from symptom onset to IVUS imaging was $1.4 \pm 1.9$ days. The interval from symptom onset to IVUS imaging might, in part, explain the differences among these studies. ${ }^{7}$ There are no currently recognized IVUS features of plaque erosion. Thus, whether nonruptured culprit lesions in AMI patients represent plaque erosion, misdiagnosis, or thrombus obscuring the rupture cavity is not clear. However, it is notable that in the present study, expansive remodeling was less frequent in nonruptured AMI lesions, a finding that has also been seen pathologically in infarct-related erosions. ${ }^{15}$

One angioscopic study reported that plaque rupture and subsequent thrombus formation were observed in $17 \%$ of SAP patients. ${ }^{16}$ In the present study, $27 \%$ of SAP patients had plaque rupture.

\section{Multiple Plaque Ruptures}

Goldstein et $\mathrm{al},{ }^{3}$ in angiography studies, Buffon et $\mathrm{al},{ }^{17}$ measuring neutrophil myeloperoxidase, and Asakura et al, ${ }^{18}$ in angioscopic studies, found multiple yellow plaques in a wide range of cardiovascular patient populations. ${ }^{12}$ In particular, Goldstein et $\mathrm{al}^{3}$ found multiple complex angiographic plaques in 100 of 253 AMI patients. Two recent IVUS studies reported the frequency of multiple plaque ruptures in ACS/ AMI patients: 79\% in the only reported 3-vessel IVUS study ${ }^{6}$ and $19 \%$ when studying just the infarct-related artery. ${ }^{19}$ Histopathological studies have classically found more than 2 plaque ruptures per AMI patient. ${ }^{2}$ This study population had primarily angiographic 1-vessel disease. Compared with Western patients, Korean patients have less extensive reference segment atherosclerosis..$^{20}$ Ruptured plaques appear to develop within the nonstenotic reference segments. These might partly explain the difference of incidence of multiple plaque ruptures between the present study (20\%) and previous studies. ${ }^{2,6,19}$ However, it is also likely that not all plaque ruptures will be detectable by IVUS, because of either size, confounding IVUS morphology (thrombus may obscure the ruptures), distal location, or branch location (in the present study, only the main vessels were imaged). The present analysis refers to IVUS-detectable plaque ruptures; a more demanding technique, such as angioscopy, would probably show more rupture, as would autopsy findings.

\section{Predictors of Plaque Rupture}

AMI was an independent overall clinical predictor of plaque rupture in patients. Compared with nondiabetic patients, patients with diabetes mellitus have a worse outcome after myocardial infarction. ${ }^{21}$ Pathological study showed that coronary atherectomy specimens from diabetic patients exhibited a larger content of lipid-rich atheroma and macrophage infiltration than specimens from nondiabetic patients. ${ }^{22}$ This is consistent with a greater probability of coronary plaque rupture in diabetic patients; in the present study, diabetes mellitus was an independent predictor of plaque rupture in SAP patients. Conversely, AMI patients with and without plaque rupture had a similar incidence of diabetes mellitus. Therefore, other factors (ie, inflammatory activity) might play a major role in plaque rupture in AMI. In AMI patients in the present study, elevated CRP level was independently associated with plaque rupture. Elevated CRP level is associated with poor prognosis in ACS and may predict future risk of AMI. ${ }^{23,24} \mathrm{~A}$ recent postmortem study showed that numbers of macrophages in thin-cap atheroma were significantly greater in patients with high CRP levels than those with low CRP levels. ${ }^{25}$ One recent IVUS study showed that the presence of ruptured plaque correlated with elevated CRP levels in AMI patients. ${ }^{14}$ However, CRP levels in the present study were measured $1.4 \pm 1.9$ days after the acute event. Thus, an elevated CRP level may be the consequence of IVUS-detectable plaque rupture in the AMI patients in the present study.

\section{Limitations}

Use of preintervention IVUS and the decision to perform 3 -vessel IVUS was at the discretion of the operator. There- 
fore, the existence of selection bias cannot be completely excluded. CRP levels were obtained just before intervention in AMI patients; thus, they may reflect the acute event and not the underlying inflammatory status of the patients. However, 71 of 122 AMI patients underwent primary stenting within 12 hours of symptom onset, and the mean duration from AMI onset to IVUS was 1.4 days.

\section{Conclusions}

Three-vessel IVUS imaging showed that culprit lesion plaque rupture (66\% versus $27 \%$ ), secondary remote plaque ruptures (17\% versus $5 \%$ ), and multiple plaque ruptures (20\% versus $6 \%$ ) were all more common in AMI patients than SAP patients. In AMI patients, plaque ruptures were more common in patients with a high CRP level, whereas in SAP patients, plaque ruptures were more common in those with diabetes. These findings may help to establish benchmarks for approaches to diagnose and treat vulnerable or ruptured plaques in patients with coronary artery disease.

\section{Acknowledgment}

This study was supported in part by the Cardiovascular Research Foundation, Seoul, Korea.

\section{References}

1. Virmani R, Kolodgie FD, Burke AP, et al. Lessons from sudden coronary death: a comprehensive morphological classification scheme for atherosclerotic lesions. Arterioscler Thromb Vasc Biol. 2000;20:1262-1275.

2. Falk E, Shah PK, Fuster V. Coronary plaque disruption. Circulation. 1995;92:657-671.

3. Goldstein JA, Demetriou D, Grines CL, et al. Multiple complex coronary plaques in patients with acute myocardial infarction. $N$ Engl $\mathrm{J}$ Med. 2000;343:915-922.

4. Maehara A, Mintz GS, Bui AB, et al. Morphologic and angiographic features of coronary plaque rupture detected by intravascular ultrasound. J Am Coll Cardiol. 2002;40:904-910.

5. Ge J, Chirillo F, Schwedtmann J, et al. Screening of ruptured plaques in patients with coronary artery disease by intravascular ultrasound. Heart. 1999;81:621-627.

6. Rioufol G, Finet G, Ginon I, et al. Multiple atherosclerotic plaque rupture in acute coronary syndrome: a 3-vessel intravascular ultrasound study. Circulation. 2002;106:804-808.

7. Kotani JI, Mintz GS, Castagna MT, et al. Intravascular ultrasound analysis of infarct-related and non-infarct-related arteries in patients who presented with an acute myocardial infarction. Circulation. 2003;107: 2889-2893.

8. Fukuda D, Kawarabayashi T, Tanaka A, et al. Lesion characteristics of acute myocardial infarction: an investigation with intravascular ultrasound. Heart. 2001;85:402-406.
9. Mintz GS, Maehara A, Bui AB, et al. Multiple versus single coronary plaque ruptures detected by intravascular ultrasound in stable and unstable angina pectoris and in acute myocardial infarction. $A m J$ Cardiol. 2003;91:1333-1335.

10. Hong MK, Park SW, Lee CW, et al. Prospective comparison of coronary artery remodeling between acute coronary syndrome and stable angina in single vessel disease: correlation between C-reactive protein and extent of arterial remodeling. Clin Cardiol. 2003;26:169-172.

11. Mintz GS, Nissen SE, Anderson WD, et al. American College of Cardiology clinical expert consensus document on standards for acquisition, measurement and reporting of intravascular ultrasound studies (IVUS): a report of the American College of Cardiology task force on clinical expert consensus documents. J Am Coll Cardiol. 2001;37:1478-1492.

12. Naghavi M, Libby P, Falk E, et al. From vulnerable plaque to vulnerable patient: a call for new definitions and risk assessment strategies: part 1. Circulation. 2003;108:1664-1672.

13. Davies MJ. A macro and micro view of coronary vascular insult in ischemic heart disease. Circulation. 1990;82(suppl II):II-38-II-46.

14. Sano T, Tanaka A, Namba M, et al. C-reactive protein and lesion morphology in patients with acute myocardial infarction. Circulation. 2003; 108:282-285.

15. Burke AP, Virmani R, Galis Z, et al. What is the pathologic basis for new atherosclerotic imaging techniques? J Am Coll Cardiol. 2003;41: $1874-1886$

16. de Feyter PJ, Ozaki Y, Baptista J, et al. Ischemia-related lesion characteristics in patients with stable or unstable angina: a study with intracoronary angioscopy and ultrasound. Circulation. 1995;92:1408-1413.

17. Buffon A, Biasucci LM, Liuzzo G, et al. Widespread coronary inflammation in unstable angina. $N$ Engl J Med. 2002;347:5-12.

18. Asakura M, Ueda Y, Yamaguchi O, et al. Extensive development of vulnerable plaques as a pan-coronary process in patients with myocardial infarction: an angioscopic study. J Am Coll Cardiol. 2001;37:1284-1288.

19. Schoenhagen P, Stone GW, Nissen SE, et al. Coronary plaque morphology and frequency of ulceration distant from culprit lesions in patients with unstable and stable presentation. Arterioscler Thromb Vasc Biol. 2003;23:1895-1900.

20. Hong MK, Mintz GS, Lee CW, et al. Intravascular ultrasound assessment of patterns of arterial remodeling in the absence of significant reference segment plaque burden in patients with coronary artery disease. $\mathrm{J} \mathrm{Am} \mathrm{Coll}$ Cardiol. 2003;42:806-810.

21. Aronson D, Rayfield EJ, Chesebro JH. Mechanisms determining course and outcome of diabetic patients who have had acute myocardial infarction. Ann Intern Med. 1997;126:296-306.

22. Moreno PR, Murcia AM, Palacios IF, et al. Coronary composition and macrophage infiltration in atherectomy specimens from patients with diabetes mellitus. Circulation. 2000;102:2180-2184.

23. Liuzzo G, Biasucci LM, Gallimore JR, et al. The prognostic value of C-reactive protein and serum amyloid A protein in severe unstable angina. N Engl J Med. 1994;331:417-424.

24. Ridker PM, Cushman M, Stampfer MJ, et al. Inflammation, aspirin, and the risk of cardiovascular disease in apparently healthy men. $N$ Engl J Med. 1997;336:973-979

25. Burke AP, Tracy RP, Kolodgie F, et al. Elevated C-reactive protein values and atherosclerosis in sudden coronary death: association with different pathologies. Circulation. 2002;105:2019-2023. 